\title{
Do spatial effects appear at low dilution rate in chemostat?
}

\section{Tatsuo Miyazaki ${ }^{1}$, Tatsuya Togashi ${ }^{1}$, Nariyuki Nakagiri ${ }^{2}$, Yukio Sakisaka $^{3}$, Kei-ichi Tainaka ${ }^{4}$ and Jin Yoshimura ${ }^{1,4,5}$,}

\author{
${ }^{1}$ Marine Biosystems Research Center, Chiba University, 1 Uchiura, Kamogawa Chiba-ken \\ 299-5502, Japan (Email: Togashi: togashi@faculty.chiba-u.jp, Miyazaki: \\ myzkt@faculty.chiba-u.jp) \\ ${ }^{2}$ School of Human Science and Environment, University of Hyogo, 1-1-12, Shinzaike- \\ honcho, Himeji 670-0092, Japan (e-mail: Nakagiri: nakagiri@shse.u-hyogo.ac.jp, tel/fax. \\ $+81-79-292-9420)$ \\ ${ }^{3}$ Center for Humanities and Sciences, Ibaraki Prefectural University of Health Sciences, \\ 4669-2 Ami, Ami-machi, Inashiki-gun, Ibaraki-ken 300-0394, Japan (email: Sakisaka: \\ sakisaka@ipu.ac.jp) \\ ${ }^{4}$ Department of Systems Engineering, Shizuoka University, Hamamatsu 432-8561, Japan \\ (e-mails: Tainaka: tainaka@sys.eng.shizuoka.ac.jp, Yoshimura: \\ jin@sys.eng.shizuoka.ac.jp) \\ ${ }_{5}^{5}$ Department of Environmental and Forest Biology, State University of New York College \\ of Environmental Science and Forestry, Syracuse, New York 13210 USA
}

*corresponding author: Jin Yoshimura jin@sys.eng.shizuoka.ac.jp 


\begin{abstract}
The chemostat theory on two species competition has shown that the dilution rate where transition of dominance occurs -transision-dilution rate- is independent of limiting-nutrient concentration. However, we obtained the experimental data indicating that the transition-dilution rate changed with variations in limiting-ammonium concentrations, using the chemostat mixed-culture of the cyanobacterium Microcystis novacekii and the green alga Scenedesmus quadricauda. The transition-dilution rate was dependent on the concentration of limiting ammonium in the influx culture medium. We tried to simulate the experimental results. Though the dilution rate has been considered independent of nutrient concentration, we introduce the effective dilution rate that depends on nutrient concentration (ammonium concentration in this study). A hyperbolic Monodtype function is used to represent the effective dilution rate for each species. The maximum dilution rate of the function is set to be the mechanical dilution rate (nominal dilution rate) of the chemostat culture. The calculation shows that the nominal transitiondilution rate where transition of dominance occur decreases with increased concentration. This simulation is well consistent with our experimental data. These results may suggest that the species-specificity of limiting nutrients, here nitrogen. Or they may imply that the depreciation of nitrogen becomes critical when both dilution rate and concentration are very low, especially for the green algae. In the latter case, spatial effects are induced internally in the ecosystem.
\end{abstract}

Keywords: Algae $\cdot$ Chemostat $\cdot$ Competition $\cdot$ Dilution rate $\cdot$ Limiting nutrient $\cdot$ Spatial effects 


\section{Introduction}

Dominant species in algal blooms is often determined by the competition between algae for resources such as nutrients. Mixed algal chemostat theory and cultures have been used as a model to study the competition (Grover, 1997). In the chemostat, the culture medium containing a limiting nutrient continuously flows into a culture vessel and the same amount of cultured algal suspension as that of influx medium flows out. Tilman (1982) applied successfully this system to elucidate phytoplankton dominance in nature.

In the chemostat system, only one species often survives and others become extinct. However, the dominating species varies, depending on the dilution rate and concentration of limiting nutrient. According to the chemostat theory, two species cultures are expected to show that changes in dominant species occurs at constant dilution rate, irrespective of concentrations of limiting nutrients (Kuwata and Miyazaki, 2000). However, it has not been examined well whether this is the case in the wide range of nutrient concentrations. There is a possibility that the dominance change depends on nutrient concentrations. If the change denpends on the concentration, there would be a need to reconsider the formulation of the chemostat theory.

In this study, we examined the competition between freshwater algae; the cyanobacterium Microcystis novacekii and the green alga Scenedesmus quadricauda, using the chemostat systems. Microcyxtis novacekii blooms often in summer in temperate lakes and deteriorates water quality (Collins, 1978; Skulbertg et al., 1984; Turner et al., 1990). The blooms usually occur in still waters. Though $S$. quadricauda is present in most lakes, it makes seldom large blooms. Using these two algae as model species may be useful in considering algal competition in the field. We measured the dominance of the two species in competition experiment, and obtained the relationship between the dilution rate and the concentration of limiting nutrient. Based on the experimental results, we assumed that the dilution rate (the volumetric flow rate / the volume of culture vessel) was a function of the nutrient concentration and deviated from constant especially under low nutrient concentrations. Calculation based on this assumption explained the change in dominance of the algae in the culture system. The function of dilution rates could be species-specific. We discuss the possible mechanisms for this species-specificity for nutrient requirements. We also discuss the possibility that the species-specificity can cause coexistence of phytoplankton under low nutrient conditions.

\section{Materials and methods}

\subsection{Competition experiment in chemostat culture}

The cyanobacterium Microcystis novacekii and the green alga Scenedesmus quadricauda were precultured in batch at room temperature at an irradiance of $20 \mu \mathrm{E} \mathrm{m}^{-2} \mathrm{~s}^{-1}$ in 14:10 LD cycles in modified WC medium (Guillard and Lorenzen, 1972). including MOPS as a buffering agent to regulate $\mathrm{pH}$ at 7.8 , and ammonium as a nitrogen source. Silicate was removed from the medium. The cells of $M$. novacekii were present in the form of unicells.

Chemostat cultures were carried out at $25^{\circ} \mathrm{C}$ and illuminated continuously at an irradiance of $150 \mu \mathrm{E} \mathrm{m}^{-2} \mathrm{~s}^{-1}$ (Watanabe and Miyazaki, 1996; Nagao and Miyazaki, 2002). Culture medium contained 10、50 and $100 \mu \mathrm{M}$ of ammonium chloride as a nitrogen source, where nitrogen limited growth of both the algae (Watanabe and Miyazaki, 1996). 
This medium was continuously supplied to the culture vessels of 1-liter at a dilution rate of $0.05,0.1$ and $0.3 \mathrm{~d}^{-1}$. M. novacekii was cultivated for $2-3$ weeks, and S. quadricauda for 3 - 5 days before the competition experiments. The cultivated cells were inoculated to the culture vessels at the densities of $10^{3} \sim 10^{4}$ cells $\cdot \mathrm{ml}^{-1}$ for $M$. novacekii and $10 \sim 10^{3}$ cells $\cdot \mathrm{ml}^{-1}$ for $S$. quadricauda. The cultures were continuously stirred with magnetic stirrers. Samples of $8 \mathrm{ml}$ were removed from the culture vessels at predetermined time and fixed with a few drops of Lugol's solution. The number of cells was counted with an inverted microscope (ZEISS Axiometer 135). A minimum of 400 cells per sample were counted. The time courses of changes of cell number were monitored for 20-35 days. Then, dominance of algae was estimated after duplicate experiments in each condition.

\subsection{Simulation}

We used the model equations based on chemostat theory (Tilman, 1982; Smith and Waltman, 1995; Kuwata and Miyazaki, 2000). Calculation was made using Mathematica 3.0.

$$
\begin{aligned}
& \frac{d Q_{m}}{d t}=V_{m}-\mu_{m} Q_{m} \\
& \frac{d Q_{s}}{d t}=V_{s}-\mu_{s} Q_{s} \\
& \frac{d S}{d t}=D\left(S_{0}-S\right)-V_{m} C_{m}-V_{s} C_{s} \\
& \frac{d C_{m}}{d t}=\left(\mu_{m}-D\right) C_{m} \\
& \frac{d C_{s}}{d t}=\left(\mu_{s}-D\right) C_{s}
\end{aligned}
$$

where $S$ is ambient ammonium concentration, $D$ is dilution rate, $V$ is ammonium uptake rate per cellular carbon, $\mu$ is specific growth rate, $Q$ is cell quota of nitrogen expressed as cellular N:C, $C$ is biomass. Subscripts $\mathrm{m}$ and s refer to $M$. novacekii and $S$. quadricauda, respectively. Equations (1) and (2) express the mass balance of intracellular nitrogen content of M. novacekii and S. quadricauda, respectively. Eq. (3) formulates the nitrogen mass balance within the culture system. Eqs. (4) and (5) show the carbon balance of the $M$. novacekii and of $S$. quadricauda in the culture system, respecitively.

Ammonium uptake rates are expressed by the Michaelis-Menten type equation (Dugdale, 1967; Legovic and Cruzado, 1997).

$$
V=V_{\max } \frac{S}{K_{u}+S}
$$

where $V_{\max }$ is the maximum ammonium uptake rate per cellular carbon and $K_{u}$ is the half saturation constant.

The relationship between growth rate $(\mu)$ versus cellular N:C, which is an alternative indicator of cell quota of nitrogen. $Q$ (Droop, 1973), is given by the linear function (Healey and Hendzel, 1988; Watanabe and Miyazaki, 1996). 


$$
\begin{aligned}
& \mu_{m}=a_{m}\left(Q_{m}-Q_{0}\right) \\
& \mu_{s}=a_{s}\left(Q_{s}-Q_{0 s}\right)
\end{aligned}
$$

where $a$ is the slope of $\mu$ versus $Q$.

To improve fitness to the experimental data, the dilution was assumed to depend on nutrient concentration. In this study, we used a Monod-type hyperbolic function:

$$
D(S)=\frac{D_{0} S}{k+S}
$$

where $D(S)$ was effective dilution rate at $S$ (concentration of limiting nutrient), $D_{0}$ is the nominal dilution rate that is a dilution rate in usual chemostat and $k$ is a constant. If $S$ becomes large enough, $D(S)$ becomes $D_{0}$ in which case the chemostat corresponds to the usual one. If $S$ is low, $D(S)$ dependency on $S$ is clearly observed (Table 2). Calculation was carried out by changing $D_{0}$ and $S$. The parameters used in this study (Table 1 ) were obtained from the culture experiments by Watanabe and Miyazaki (1996).

\section{Results}

\subsection{Culture experiments}

Figure 1 shows the time-series changes of cell densities in mixed chemostat culture of $M$. novacekii and Scenedesmus quadricauda at $0.1 \mathrm{~d}^{-1}$ of dilution rate and at $10 \mu \mathrm{M}$ of ammonium. In this case, $M$. novacekii expelled $S$. quadricauda from the culture medium. In this way, we determined dominant species in the mixed cultures at different sets of dilution rates and concentrations of limiting nutrient (ammonium).

Dominance of M. novacekii or S. quadricauda was shown in Table 2 and Fig.2. The dilution rate where transition of dominance occurred changed, according to limiting ammonium concentration in the supply medium. At $10 \mu \mathrm{M}$ of ammonium, the transitiondilution rate was between 0.1 and $0.3 \mathrm{~d}^{-1}$. At $50 \mu \mathrm{M}$, the transition-dilution rate seemed less than $0.1 \mathrm{~d}^{-1}$. At $100 \mu \mathrm{M}$, the transition-dilution rate was between 0.05 and $0.1 \mathrm{~d}^{-1}$. Figure 2 shows that the transition line of dominance is round at lower dilution rates and ammonium concentrations. According to the previous chemostat theory, dilution rate is not a function of nutrient concentration (Kuwata and Miyazaki, 2000). Therefore, the dominance transition should be constant at between 0.05 and $0.1 \mathrm{~d}^{-1}$, irrespective of ammonium concentration (10$100 \mu \mathrm{M}$ ). However, the current result (Fig. 2) shows that dominance transition also appear when the ammonium concentration is varied between $50 \mu \mathrm{M}$ and $10 \mu \mathrm{M}$ at a constant dilution rate of $0.1 \mathrm{~d}^{-1}$.

\subsection{Simulation}

We calculated the time courses of population density using the effective dilution rate Eq. (9) that depends on nutrient concentration. Figure 3 shows that time courses at $0.3 \mu \mathrm{M}$ of ammonium. M. novacekii dominates at lower dilution rates of $0.01 \mathrm{~d}^{-1}$, whereas $S$. 
quadricauda dominated at a higher dilution rate of $0.95 \mathrm{~d}^{-1}$. The transition- dilution rate where dominance changed was obtained from these time courses. Table 3 and Fig. 4 exhibit the nominal dilution rate at which dominance-transition occurred. At $0.1 \mu \mathrm{M}$ of ammonium the dominance change occurred at the nominal dilution rate of $0.86 \mathrm{~d}^{-1}$. At 100 $\mu \mathrm{M}$, it occurred at $0.08 \mathrm{~d}^{-1}$. This indicates that dominance change occurs at different dilution rates at the different limiting nutrient concentration, and corresponds to the experimental results.

\section{DISCUSSION}

The present culture experiments suggest the dilution and the concentration of limiting nutrient were mutually dependent. The lower dilution and lower nutrient concentration favored the dominance of $M$. novacekii, compared with $S$. quadricauda. In natural waters, turnover of water was usually smaller than that in chemostat culture experiments, and the concentration of ammonium was less than several $\mu \mathrm{M}$. Thus, it may be necessary to consider the interdependency of nutrient concentration and dilution when considering algal blooms and competition in natural waters.

We used, as a first-order approximation, the Monod-type function for the expressing the relationship between dilution rate and limiting-nutrient concentration (Tilman, 1982), though other types of function might be possible. This introduction of the Monod-type function into chemostat equations explained the results of algal cultures in the present study. At lower concentration of limiting ammonium, dominance transition occurred at lower nominal dilution rates.

The equation (9) indicates that effective dilution rates are lower than nominal ones at lower concentrations of limiting nutrient. The parameter $k$ of the equation (9) can be species-specific. If so, effective dilution might be also species-specific. Then, turnover of each species could be different even in the same nominal dilution. In this situation, more than one species could coexist under the same dilution of water in the apparently homogeneous environments, since the equilibrium might be different for each species.

Theoretical predictions state that only one species survive in a homogeneous environment (see Grover 1997). In the chemostat system of one limiting nutrient, only one species survives and others become extinct, as suggested by the theory. If more than two nutrients are limiting the growth of organisms, coexistence may become possible (Tilman 1962). Grower (1997) suggested that the maximum number of coexisting species is the number of limiting nutrients.

In our system, at lower concentrations of limiting ammonium, nominal dilution rates were more different from effective dilution rates. Furthermore, $k$ in the equation (9) can be species-specific. If so, more than one algal species in homogeneous environments could coexist at lower concentrations of a limiting nutrient, because each species with different dependency of dilution on nutrient concentration (9) would reach the different equilibrium. Here the species-specificity in the limiting nutrients is considered a type of resource segregation that is similar to habitat segregation. Thus the number of limiting nutrients may explain the coexistence of a few phytoplankton species. 
However, many more species coexist in natural waters than the numbers of limiting nutrients that is usually a few (Hutchinson 1961). He called the coexistence in apparently homogenous water environment, "the paradox of plankton." Thus we have no solid explanation for the coexistence of many species of phytoplankton in apparently homogeneous water environments.

One way to explain this paradox of environment is external disturbance to water environments. Disturbance often create the heterogeneity in the water environment, resulting in the opportunities for the coexistence of different species. In the chemostat, Tilman (1962) suggested that fluctuation of nutrient inflow might yield the heterogeneity of the chemostat, resulting in the coexistence of phytoplankton. Similarly, external disturbances may be caused by temporal perturbation, inhomogeneousness of space, or predation (Richerson et al., 1970; Levins, 1979; Sommer, 1985; Padisak et al., 1993). These explanations imply the apparent homogeneous environment is not really homogeneous, but heterogeneous with respect to some environmental factors.

Contrary, Huisman and Welssing (1999) suggested that an external factor may not be necessary for the coexistence of species. They showed theoretically that the diversity might be explained without considering external perturbation. In their resource competition model, the competition itself could generate oscillations and chaos in a spatial ecosystem. In our system, spatial effects in dilution and/or concentrations may also explain the coexistence. In this case, concentrations of a limiting nutrient (i.e., nitrogen) may be insufficient for the cells of $S$. quadricauda in their vicinity, resulting in a heterogeneous environment. This is also an internally induced heterogeneity of environment. However, these explanations can only explain the coexistence of several species, but not of hundreds of species.

Another possibility for coexistence in our system is the avoidance of competition in extremely low nutrient concentrations resulting from very low growth rates of both species. At higher concentrations algae can grow enough at lower nominal dilution since nutrient deprivation did not occur due to higher nutrient concentrations. Here nominal dilution rates are almost the same as effective ones. At very low dilution rate and/or very low nutrient concentrations, the growths of both species are reduced at very low rates, resulting in extremely low densities. Therefore, the cells of both species are separated widely and the competition for resources does not occur between the species (Miyazaki et al. 2006). This is another internal mechanism for the coexistence of phytoplankton in a homogeneous environment. This mechanism may explain the extremely many species of coexistence, i.e., the paradox of plankton (Hutchinson 1961), in oligotrophic waters (Leibold, 1999).

Acknowledgments The authors are grateful to Mr. K. Takeya and Dr. A. Kuwata for their valuable help in experiments and simulation. They also wishes to thank Dr. P.A. Cox for his comments on the manuscript. 


\section{References}

Collins, M., 1978. Algal toxins. Micorbiol. Rev. 42, 725-746.

Droop, M.R., 1973. Some thoughts on nutrient limitation in algae. J. Phycol. 9, 264-272.

Dugdale, R.C., 1967. Nutrient limitation in the sea: Dynamics, identification and significance. Limnol. Oceanogr. 12, 685-695.

Galliard, R.R.L., Lorenzen, C.J., 1972. Yellow-green algae with chlorophyll c. J. Phycol. 8, 10-14.

Grover, J.P., 1997. Resource Competition. Chapman \& Hall, London.

Healey, F.P., Hendzel, L.L., 1988. Competition for phosphate between desmids. Limnol. Oceanogr. 26, 622-634.

Huisman, J., Welssing, F.J., 1999. Biodiversity of plankton by species oscillations and chaos. Nature 402, 407-410.

Hutchinson, G.E., 1961. The paradox of plankton. Am. Nat. 95, 137-145.

Kuwata, A., Miyazaki, T., 2000. Effects of ammonium supply rates on competition between Microcystis novacekii (Cyanobacteria) and Scenedesmus quadricauda (Chlorophyta): simulation study. Eco. Mod. 135, 81-87.

Legovic, T., Cruzado, A., 1997. A model of phytoplankton growth on multiple nutrients based on the Michaelis-Menten-Monod uptake. Droop's growth and Liebig's law. Eco. Mod. 99, 19-31.

Leibold, M.A., 1999. Biodiversity and nutrient enrichment in pond plankton communities. Evol. Ecol. Res. 1, 73-95.

Levins, R., 1979. Coexistence in a variable environment. Am. Nat. 114, 765-783.

Miyazaki, T., Tainaka, K., Togashi, T., Suzuki, T., Yoshimura, J., 2006. Spatial coexistence of phytoplankton species in ecological timescale. Population Ecology 48, 107-112.

Nagao, M., Miyazaki, T., 2002, Release of dissolved organic nitrogen from Scenedesmus quadricauda (Chlorophyta) and Microcystis novacekii (Cyanobacteria). Aquat. Microb. Ecol. 27, 276-284.

Padisak, J., Reynolds, C.S., Sommer, U., 1993, The intermediate disturbance hypothesis in phytoplankton ecology. Hydrobiologia 249, 1-199.

Richerson, P.J., Armstrong, R., Goldman, C.R., 1970. Contemporaneous disequilibrium: a new hypothesis to explain the paradox of plankton. Proc. Natl. Acad. Sci. USA 67, 1710-1714.

Skulberg, O.M., Codd, G.A., Garmichael, W.W., 1984, Toxic blue-green algal blooms in Europe: a growing problem. Ambio, 13, 244-247.

Smith, H.L., Waltman, P., 1995. The Theory of the Chemostat. Cambridge University Press, New York.

Sommer, U., 1985. Comparison between steady state and non-steady state competition: experiments with natural phytoplankton. Limnol. Oceanogr. 30, 335-346. 
Tilman, D., 1982. Resource competition and community structure. Princeton University Press, Princeton.

Turner, P.C., Gammie, A.J., Hollinrake, K., Codd, G.A., 1990. Pneumonia associated with contact with cyanobacterium. Br. Med. J. 300, 1440-1441.

Watanabe, T., Miyazaki, T., 1996. Maximum ammonium uptake rates of Scenedesmus quadricauda (Chlorophyta) and Microcystis novacekii (Cyanobacteria) grown under nitrogen limitation and implications for competition. J. Phycol. 32, 243-249. 
Table 1 - Parameters for calculation used for the present study.

\begin{tabular}{llll}
\hline Parameter & M.novacekii & S. quadricauda & Unit \\
\hline$V_{\max }$ & 0.206 & 0.696 & $\mathrm{~mol} \mathrm{~N} \mathrm{~mol} \mathrm{C} \mathrm{d}_{-1}$ \\
$K_{u}$ & 0.5 & 1.25 & $\mu \mathrm{M}$ \\
$a$ & 6.33 & 32.26 & $\mathrm{~mol} \mathrm{~N}^{-1} \mathrm{~mol} \mathrm{C} \mathrm{d}^{-1}$ \\
$Q_{0}$ & 0.0237 & 0.465 & $\mathrm{~mol} \mathrm{M} \mathrm{mol} \mathrm{C}$ \\
$k$ & 1 & 1 & $\mu \mathrm{M}$ \\
\hline
\end{tabular}


Table 2 - Dominat species in two species mixed culture Scenedesmus quadricauda and Microcystis novacekii under different sets of dilution rate and ammonium concentration in supply medium. Cultures were carried out at $25^{\circ} \mathrm{C}$.

\begin{tabular}{llll}
\hline $\begin{array}{l}\text { Ammonium } \\
\text { concentrations in } \\
\text { supply medium }\end{array}$ & $0.05 \mathrm{~d}^{-1}$ & Dilution rate & \\
\cline { 2 - 4 } & & $0.1 \mathrm{~d}^{-1}$ & $0.3 \mathrm{~d}^{-1}$ \\
\hline $10 \mu \mathrm{M}$ & M. novacekii & S. quadricauda \\
$50 \mu \mathrm{M}$ & M. novacekii & S. quadricauda & \\
$100 \mu \mathrm{M}$ & S. quadricauda & \\
\hline
\end{tabular}


Table 3 - Some calculated transitiondilution rate vs. ammonium concentration in the supply medium.

\begin{tabular}{ll}
\hline $\begin{array}{l}\text { Ammonium } \\
\text { concentration } \mu \mathrm{M}\end{array}$ & $\begin{array}{l}\text { Transition-dilution rate } \\
\text { (nominal) } \mathrm{d}^{-1}\end{array}$ \\
\hline 0.1 & 0.86 \\
0.5 & 0.24 \\
5 & 0.095 \\
50 & 0.08 \\
100 & 0.08 \\
\hline
\end{tabular}


Figure legends

Fig. 1 - Changes in cell densities of $M$. novacekii (circles with solid line)and $S$. quadricauda (triangles with broken line) at 0.1 day $^{-1}$ of dilution rate and $10 \mu \mathrm{M}$ of ammonium.

Fig. 2 - Supposed transition curve in chemostat with two competing species $M$. novacekii (circles) and $S$. quadricauda (triangles). The dominant species are plotted from the experimental results (Table 2$)$. When the dilution rate is less than $0.1\left(\right.$ day $\left.^{-1}\right), M$. novacekii win over S. quadricauda.

Fig. 3 - Time courses of biomass changes at $0.3 \mu \mathrm{M}$ of ammonium in similation. We can detect the dominance-transition parameter sets (dilution rate and ammonium concentration) from these graphs when the biomass of both species (M. novacekii: solid curve; $S$. quadricauda: broken curve) keep steady state in later days (in the graph both curves form parallel lines). a) dilution rate $=0.01$ day $^{-1}$. b) dilution rate $=0.95$ day $^{-1}$.

Fig. 4 - Theoretical transition-dilution rate versus ammonium concentration in the supply medium. Each circle represents a coexisting point calculated from simulations as Fig. 3. Solid curve is drawn by spline interpolation of all plots. In the inserted figure, the log scale is used in y-axis to show the transition at low ammonium concentrations. 


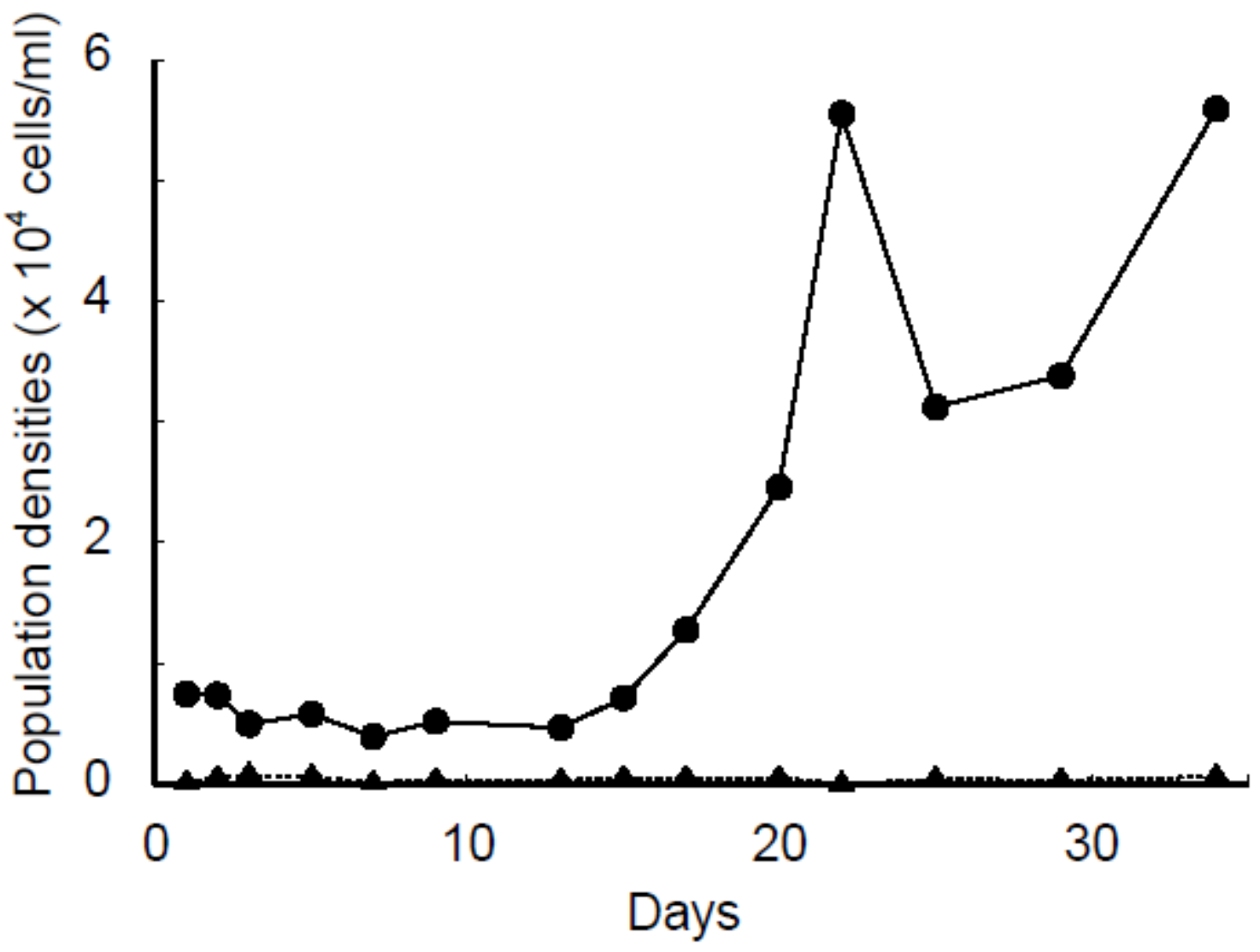

Fig. 1 


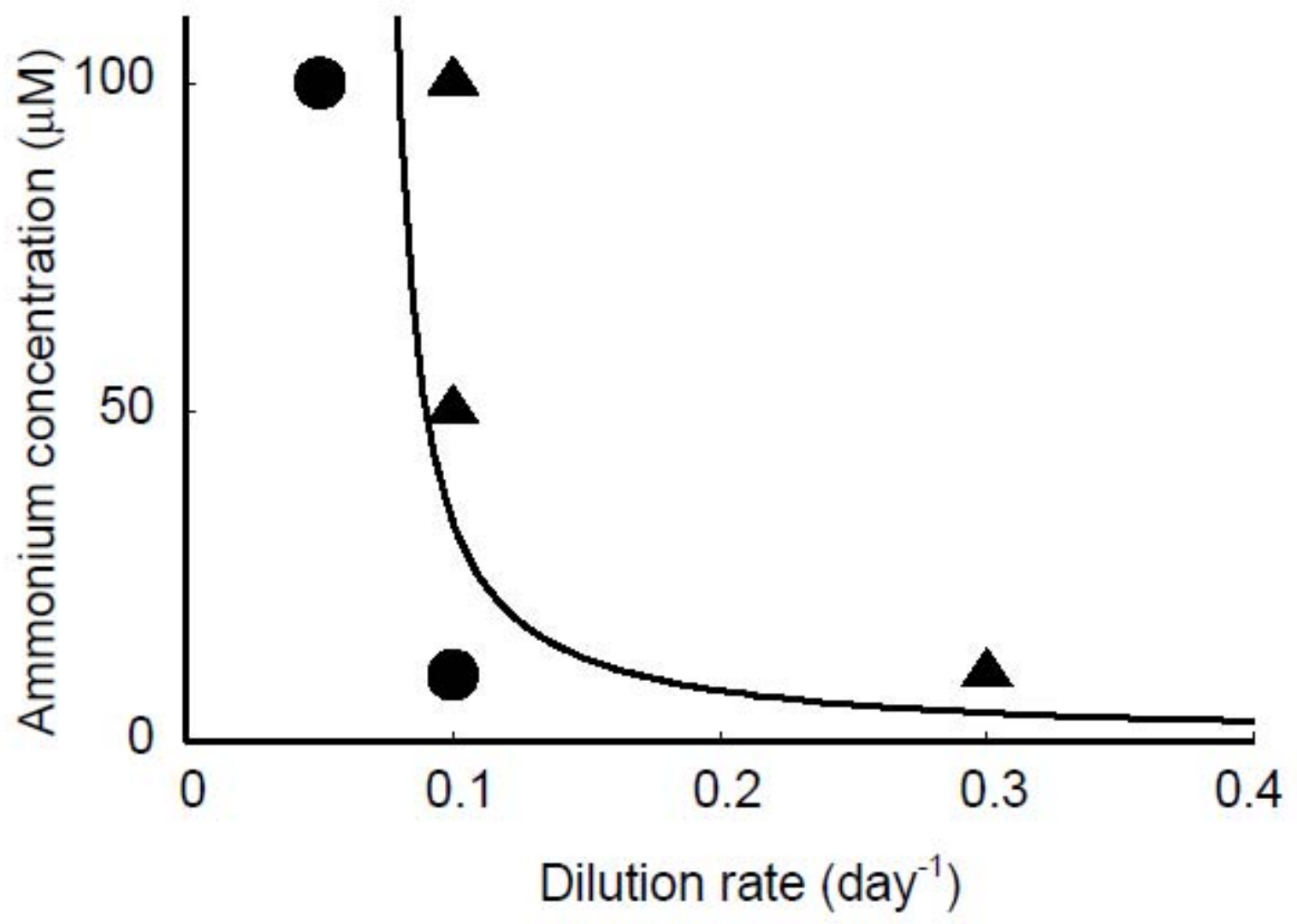

Fig. 2 


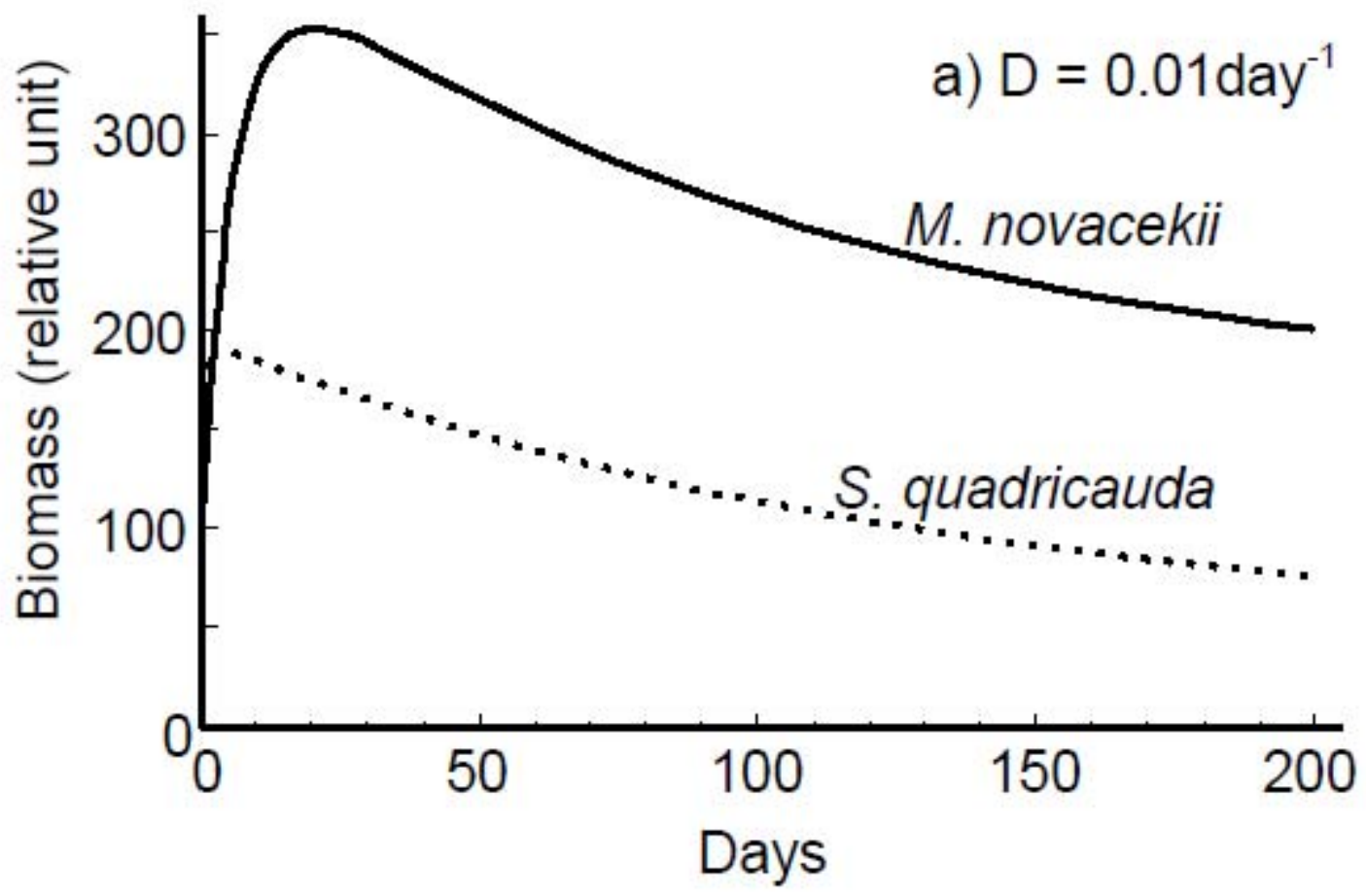

Fig. 3a 


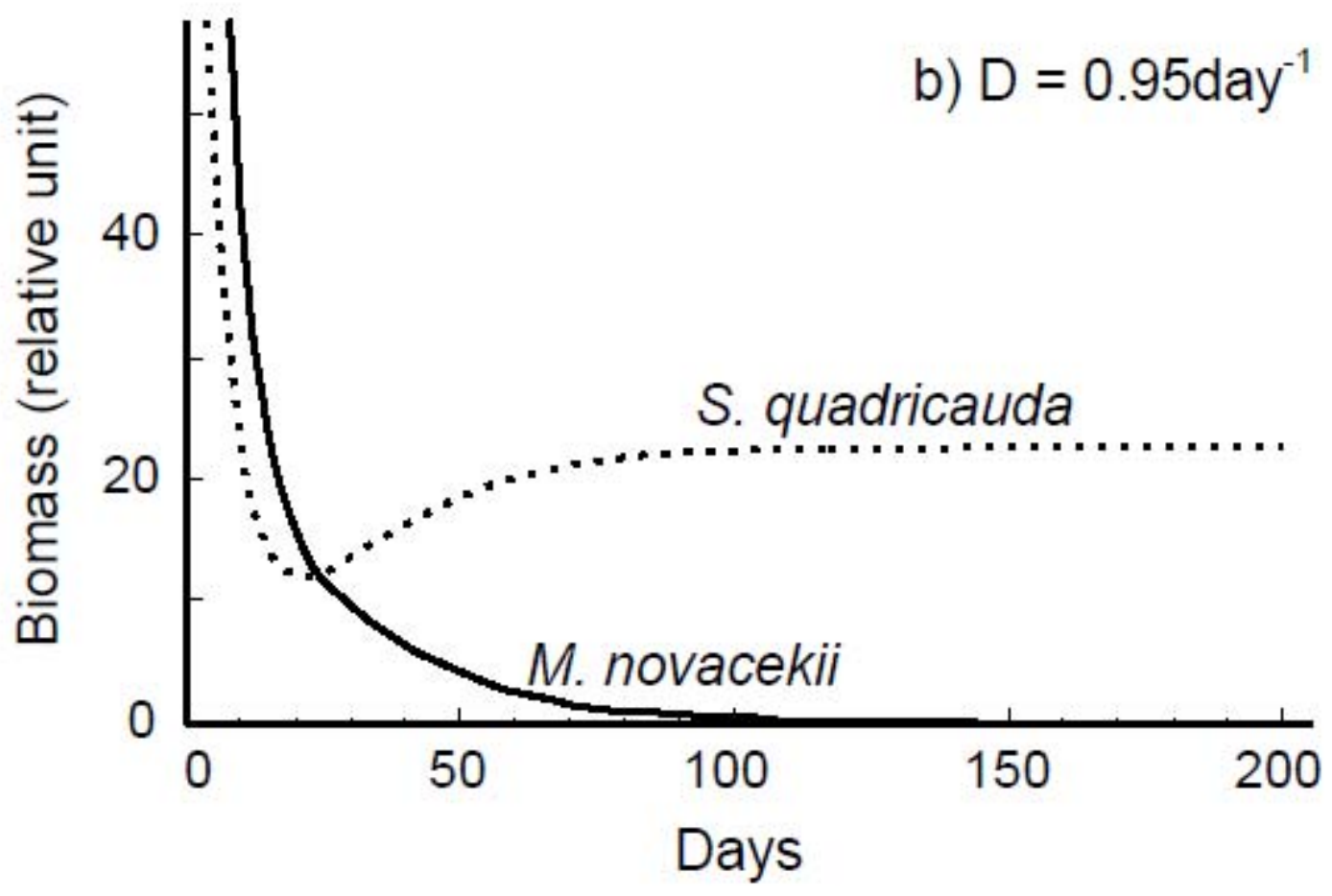

Fig. $3 b$ 


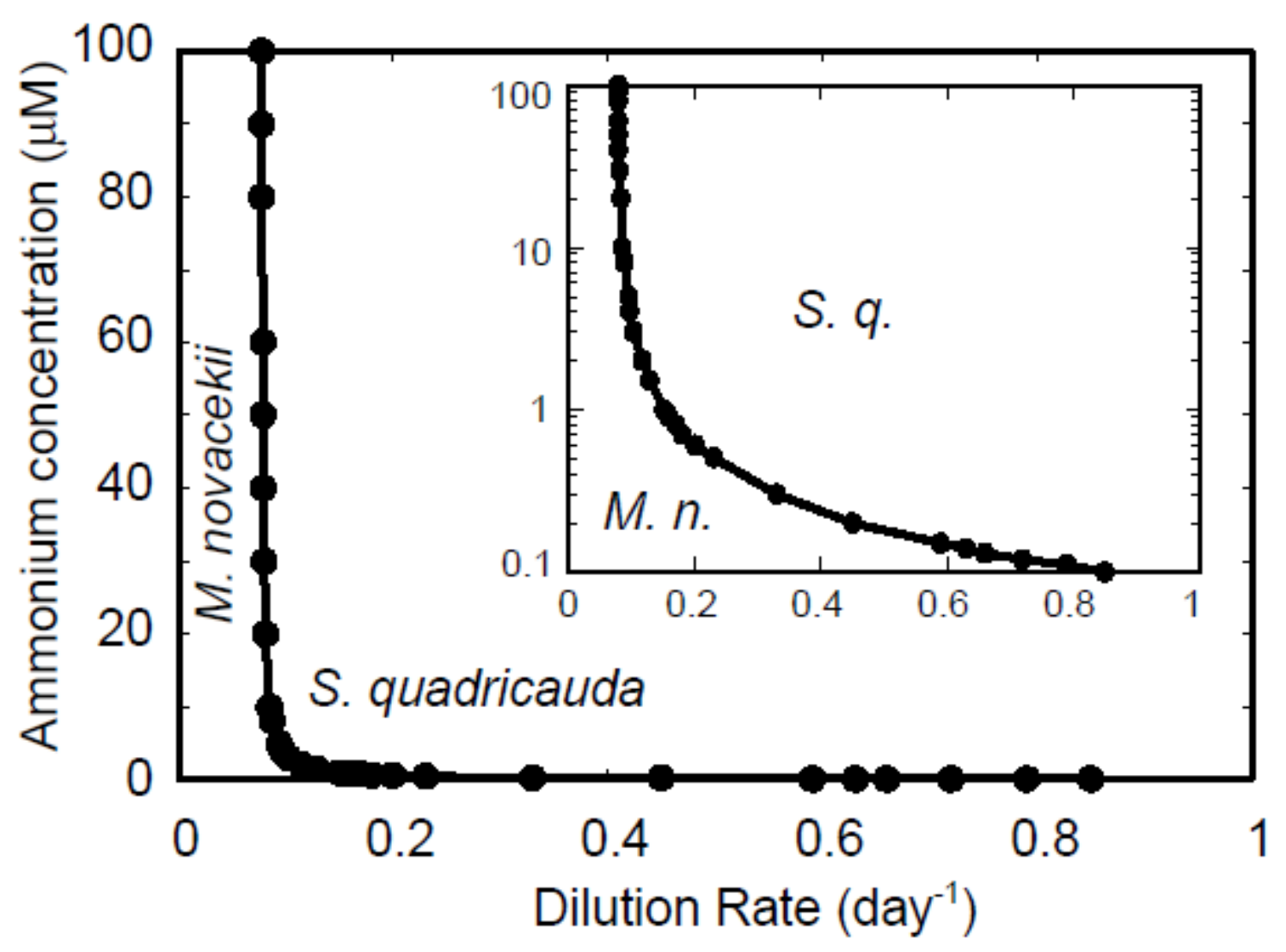

Fig. 4 